\title{
High positive computed tomography yields in the emergency department might not be a positive finding
}

\author{
K Swartzberg, MB ChB, DipEC (SA); L N Goldstein, MB BCh, MMed, FCEM (SA), Cert Critical Care (SA) \\ Division of Emergency Medicine, Faculty of Health Sciences, University of the Witwatersrand, Johannesburg, South Africa
}

Corresponding author: K Swartzberg (kylen.swartzberg@gmail.com)

\begin{abstract}
Background. There is growing pressure to reduce unnecessary computed tomography (CT) imaging requests that the radiology department receives from the emergency department (ED); however, information on acceptable usage rates and diagnostic yields remains scanty. Objectives. To describe the indications, clinical categories and positive yield rates of patients receiving CT scans in the ED. Methods. A retrospective record review was done of all patients who received CT scans at an urban, adult academic ED during a 4-month period. Primary outcomes were to establish CT scan usage and positive yield rates. Other outcomes included analysis of indications, demographics and anatomical areas scanned.

Results. Scans ( $n=1010)$ were analysed. The median age of patients was 36 (range 4 - 93) years. Male patients received $64.3 \%$ of all scans, as well as $75.7 \%$ of the scans performed for trauma. The majority of the scans were for trauma patients. However, non-trauma patients had a higher positive yield; the non-trauma positive yield rate was $61.8 \%$ compared with the trauma positive yield rate of $47.1 \%(p<0.001)$. The majority of scans performed were of the head (58\%) and neck (20\%), with lowest positive yield rates of $48.9 \%$ and $17 \%$, respectively. The overall CT scan usage rate was $4.6 \%$ and overall positive rate $53.8 \%$.

Conclusion. A negative CT scan does not necessarily mean that the test was not indicated. Higher positive yield rates may reflect insufficient use of CT scanning by the ED. Local guidelines should be established to ensure judicious and effective clinical use of CT scans.
\end{abstract}

S Afr Med J 2018;108(3):230-234. DOI:10.7196/SAMJ.2018.v108i3.12635

A computed tomography (CT) scan is an effective imaging tool that can identify many disease processes and alter the management of patients who present to the emergency department (ED), especially when the diagnosis is uncertain. ${ }^{[1,2]}$ It is therefore an invaluable diagnostic tool in emergency medicine, which is evidenced by a substantial increase in the number of CT scans requested by doctors working in the ED. ${ }^{[3]}$

In an analysis of licensed South African (SA) diagnostic imaging equipment, there were only 5 CT scanners per 1 million general population compared with 101 scanners per 1 million population in Japan and almost 41 per 1 million in the USA. ${ }^{[4]}$ In a country where 44 million people are dependent on public health resources, the effective and judicious use of CT scanners needs to be scrutinised. ${ }^{[4]}$

ED doctors are required to correctly manage patients in a timely fashion. They need to rapidly and accurately diagnose and decide on a patient's disposition, while making use of the least invasive modality of diagnostic testing. ${ }^{[5,6]}$ In the ED setting, providers may tend to overutilise CT scans. ${ }^{[2,7]}$ Other factors contributing to CT over-utilisation may include fear of a missed or incorrect diagnosis, the potential for malpractice lawsuits, as well as inadequate knowledge and awareness of radiation exposure and potential risk of inducing cancer. ${ }^{[7]}$

There is an abundance of studies evaluating trends in CT scan use in EDs, with growing pressure to measure and reduce unnecessary imaging in that environment. ${ }^{[8]}$ However, studies describing usage and yield rates remain scanty. Some of these analyses have demonstrated a trend towards decreasing diagnostic yields (defined as the proportion of positive tests among all tests ordered).$^{[8-10]}$

To the best of our knowledge, there is no guidance for yield rates of emergent CT scan use in southern Africa and no acceptable standards have been set. Therefore, it is important to study the utilisation of CT scanning in the ED and to establish acceptable rates of positive yields to monitor the use of this valuable resource.
The objective of this study was to describe the types of CT scans ordered, the indications and clinical categories, to establish the positive rates compared with international rates, and to describe the patients who received CT scans in an ED in SA.

\section{Methods}

This was a retrospective record review of all patients who received CT scans requested by the ED from 1 January to 30 April 2015. The study was performed at an adult, urban tertiary academic ED in the public sector of one of SA's major metropolitan areas.

The ED receives 65000 patients annually, of whom $\sim 23 \%$ are trauma patients. All gynaecological and paediatrics services are rendered at a nearby sister hospital. The radiology department performs an average of $700 \mathrm{CT}$ scans per month; these include emergent and non-emergent scans.

All scans that were requested and performed on patients from the ED during the abovementioned period, were used for the study.

Exclusion criteria included reports not filed or not found, and illegible information.

Findings were deemed positive (a scan with a radiologically significant lesion related to the indication for the scan) or negative (no radiologically detected lesion) by the primary assessor, an emergency medicine registrar. Any findings that were ambiguous with regard to their clinical significance were reviewed by an emergency physician and a radiologist to reach consensus on the clinical relevance of the findings. A negative radiological scan is not necessarily 'negative', e.g. an acute ischaemic stroke was indicated as a negative radiological scan, but could lead to important therapeutic decisions, such as initiating thrombolysis.

The primary outcomes were to establish the positive rate or yield and to compare this with standard international expectations.

Other outcomes included analysis of indications for scans and demographics of patients receiving scans, as these have immediate 
and long-term implications on patient risk v. benefit, as well as the anatomical areas scanned and their findings, which are relevant with regard to auditing the use of clinical decision rules.

Patient characteristics and scan results were evaluated using appropriate statistics to create descriptive summaries. Analysis was performed using Microsoft Excel 2010 (Microsoft, USA) and R Core Team (a language and environment for statistical computing) (R Foundation for Statistical Computing, Austria).

\section{Ethical approval}

Ethical approval was granted by the Human Research Ethics Committee, University of the Witwatersrand, Johannesburg (ref. no. M160234), as well as by the hospital ethics committee (ref. no. 1494162).

\section{Results}

Reports ( $n=1010)$ were collected and analysed; 4 were excluded. Scans ordered from the ED constituted $36 \%$ of the total monthly scans performed in the hospital.

The minimum and maximum ages of the patients were 4 and 93 (median 36) years, respectively.

Fig. 1 shows the breakdown of the number of trauma and nontrauma scans performed in each age category. There were 25 reports where ages were omitted owing to various reasons. These have not been included in the figure. Fig. 2 shows the total number of positive scans in each age category. Fig. 3 represents the percentage of each anatomical region scanned overall.

In Table 1 the division of scans for trauma and non-trauma patients, as well as those performed for blunt and penetrating trauma, are set out. Table 2 indicates the total positive yield of scans in each category.

Of the 1010 patients who received CT scans during the abovementioned period, $\geq 2$ anatomical regions were scanned in 198 cases, equating to 1188 anatomical areas being scanned. Only 7 of 576 patients received full-body scans for trauma. The CT scan usage rate (total number of ED patients being sent for a scan) was $4.6 \%$ and the overall positive yield was $53.8 \%$. Fig. 4 represents the positive yield for each anatomical area scanned. Of the 29 blunt trauma abdominal scans that were performed, 19 (65.5\%) were positive. Of 361 blunt trauma head CT scans performed, there were positive findings in 163 $(45.15 \%)$ cases.

\section{Discussion}

This study is the first of its kind in SA, where no guidelines with regard to acceptable usage rates and positive yields exist, which have been found to be on par with international figures.

SA EDs experience one of the highest trauma loads in the world. In 1999, the SA Medical Research Council estimated that 1.5 million trauma patients presented to secondary and tertiary hospitals. ${ }^{[1]}$ This resulted in the investigation of trauma-related scans of particular importance in our setting.

Trauma contributed to $23 \%$ of all patient presentations to our ED. However, $57 \%$ of all scans were performed on trauma patients. It is evident that there is a high ratio of scans being ordered for patients presenting with trauma. It was anticipated at the outset that the majority of trauma scans would be for blunt trauma, which was confirmed.

As expected, more male patients were scanned owing to their significantly higher presentation with trauma. ${ }^{[12,13]}$ This was accompanied by a higher positive yield rate. Young males have a $27 \%$ higher risk of dying from trauma than females because of more severe mechanisms of trauma. Female patients often present to the ED with low-energy trauma. ${ }^{[13]}$ However, when females do present with blunt

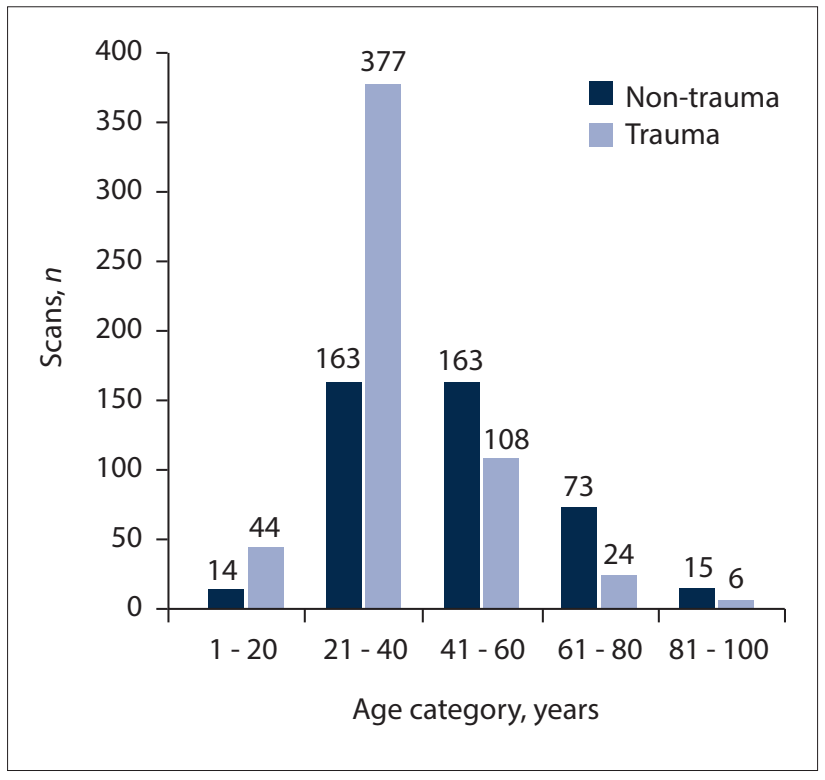

Fig. 1. Number of non-trauma and trauma scans performed in each age category.

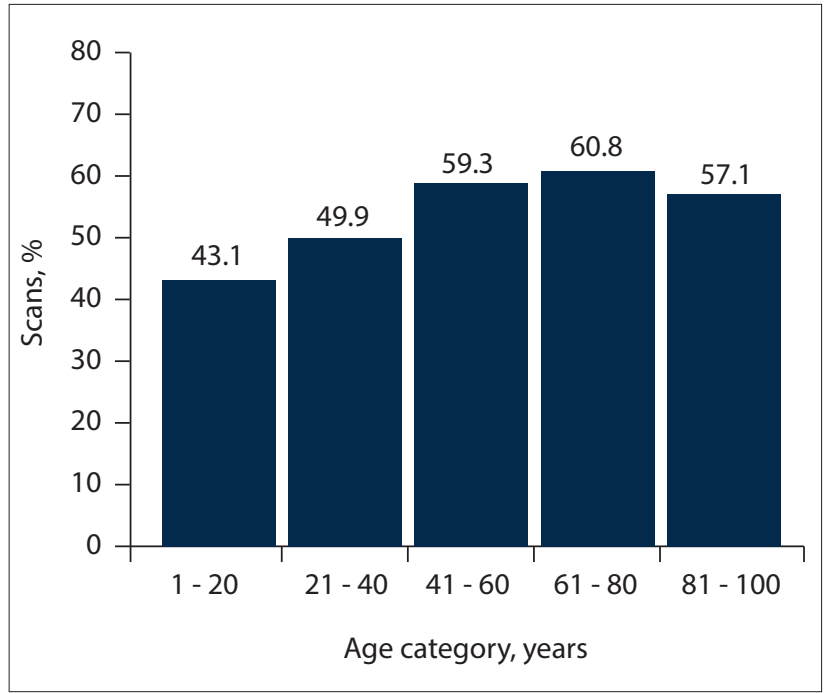

Fig. 2. Percentage of positive scans according to age category.

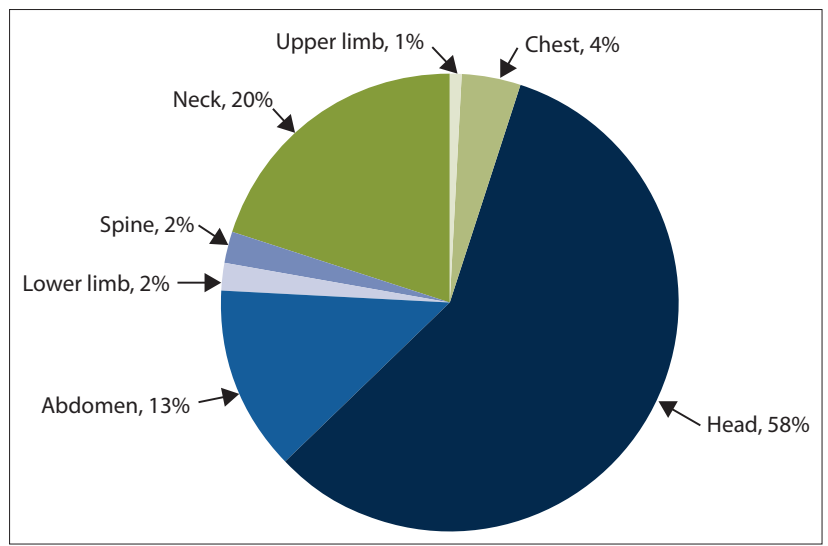

Fig. 3. Division of anatomy regions scanned.

trauma, they mainly present with headache, neck pain and back pain. ${ }^{[13]}$ In a situation where there has been a low mechanism of injury but persistent complaints of pain, providers may be obligated to perform imaging, which may then contribute to a negative scan. 
Table 1. Trauma, non-trauma, blunt and penetrating trauma according to sex

\begin{tabular}{lllc}
\hline & Male, $\boldsymbol{n}(\%)$ & Female, $\boldsymbol{n}(\%)$ & Total, $\boldsymbol{n}(\%)$ \\
\hline Trauma & $436(75.7)$ & $140(24.3)$ & $576(57.0)$ \\
Non-trauma & $213(49.0)$ & $221(51.0)$ & $434(43.0)$ \\
Blunt trauma & $339(72.3)$ & $130(27.7)$ & $469(81.3)$ \\
Penetrating trauma & $98(90.7)$ & $10(9.3)$ & $108(18.7)$
\end{tabular}

\section{Table 2. Positive yield rates in various categories}

\begin{tabular}{lll}
\hline Category & Positive yield, $\boldsymbol{n}(\%)$ & $\boldsymbol{p}$-value \\
\hline Male & $355(54.5)$ & \\
Female & $186(51.5)$ & $<0.001$ \\
Non-trauma & $269(61.8)$ & \\
Trauma & $272(47.1)$ & $<0.001$ \\
Penetrating trauma & $58(54.2)$ & \\
Blunt trauma & $214(45.7)$ & 0.138
\end{tabular}

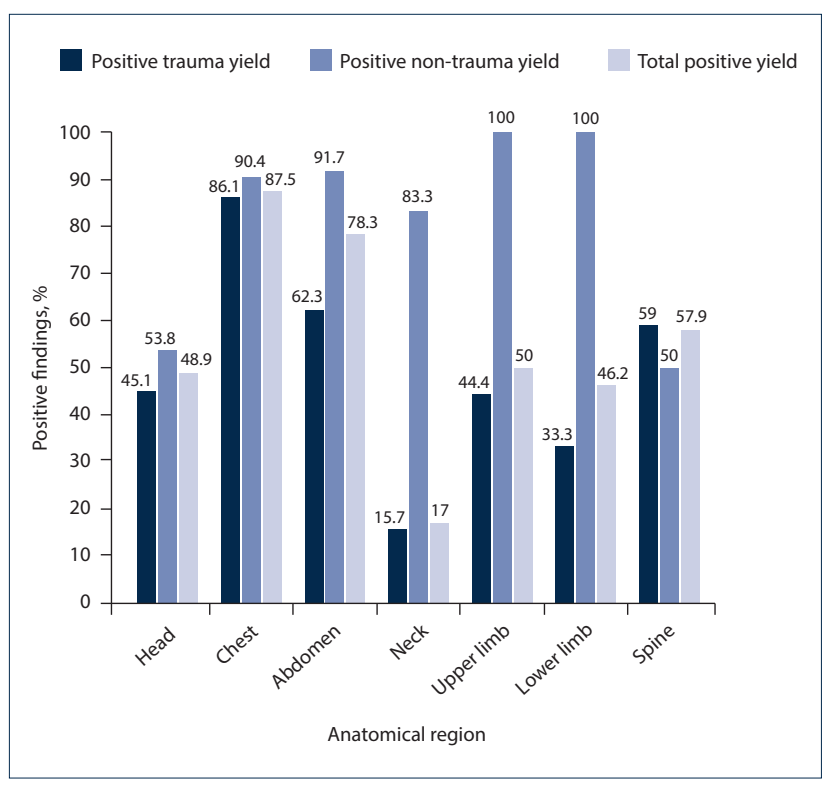

Fig. 4. Percentage of positive findings according to anatomical region scanned.

Emergency physicians rely on the results of CT scans to make decisions regarding the further management and disposition of patients. Some of these decisions lead to immediate surgical intervention. Many patients who would have obvious positive findings on a CT scan are not scanned, but instead, for various clinical reasons, are taken directly to the operating theatre. This factor may influence the positive yield of scans, as patients being considered for surgical intervention may have a negative scan and do not need to be operated on. With a cost of $\sim$ ZAR126 000 for a patient who receives emergent surgery, a negative CT scan may be cost-saving, as this will avoid the cost and possible negative sequelae of surgery. ${ }^{[14]}$ However, a positive scan may assist the surgeon and result in a better outcome for the patient.

In the literature, there is a large discrepancy between trauma and non-trauma patient CT positive yield rates. ${ }^{[1]}$ This is confirmed by the findings of this study, with positive yield rates of $47.2 \%$ and $61.8 \%$, respectively. A suggested significant contributor to these findings may be that many trauma patients undergo a full-body scan when they have a significant mechanism of injury. ${ }^{[1]}$ However, in this study of 576 trauma scans, only 7 full-body or pan scans were performed.
We maintain that there is a place for selecting certain anatomical regions for scanning in polytrauma patients, based on clinical findings, point-of-care ultrasound findings and implementation of clinical decision rules. It is evident that there is no difference in mortality with regard to patients undergoing immediate full-body scans v. those receiving selective anatomical scans. ${ }^{[15]}$ This would require further exploration in our setting.

The overall positive rate in the study was $53.8 \%$, which is higher than that in a study by Rigsby and Pai, ${ }^{[1]}$ which is one of the only studies presenting an overall positive rate $(40 \%)$. However, their study only investigated a combination of abdominal and pelvic CT scan findings. Our study had a rate of $78 \%$ for the same combination of CT scans.

In Kirschner et al.' ${ }^{[16]}$ study, with a similar setting to our study, they indicated a usage rate of $7.8 \%$ (usage rate is defined as the number of patients being sent for a scan out of the total number of patients presenting to the ED). In an ED with $>5000$ patient visits per month, only $4.6 \%$ were sent for CT scans. Factors contributing to the low rate of CT scans are clear clinical decision rules and use of point-of-care ultrasound, which impact decision-making and diagnostic work-up of patients. ${ }^{[17,18]}$ This is another important indicator of CT scan use, confirmed by many studies that show lower rates of use equating to higher positive detection rates. ${ }^{[8-10,19]}$ As our CT use rate is lower than that of other EDs, our ED might be performing too few CT scans.

As there is a lack of published material on total ED CT scan yield rates, this study had to concentrate on anatomical areas to find standards for comparison. In so doing, it was found that there are wide ranges of positive findings reported and no standards set. As an example, studies of positive rates of non-trauma head CTs found abnormalities ranging from $10 \%$ to $39 \%$; trauma head CT rates were $\sim 29 \% .{ }^{[20-22]}$ For non-trauma and trauma head scans, abnormal findings in this study were $53.8 \%$ and $45.1 \%$, respectively, which were also higher than most. A study done in Malaysia reported a positive rate of $70.5 \%$. With such wide ranges it is clear that there is need for a standard to be set or improvement of implementation of protocols. ${ }^{[23]}$

There was a significant difference in the positive yield in trauma and non-trauma CT scans of the limbs. Both upper and lower limb non-trauma scans had a $100 \%$ positive yield compared with trauma scans, which had positive yields of $44 \%$ and $33 \%$, respectively. The following may account for this difference: with trauma presentations there may be a variety of ways in which injury can present, from fractures, compartment syndrome to obvious open vascular injuries. Many of these patients are taken directly to theatre and therefore do not undergo radiological investigation. In a recently published study by Monazzam et al. ${ }^{[2]}$ on CT angiograms of fractured lower extremities, they found no evidence to support the routine use of CT angiograms to evaluate lower-extremity fractures unless at least one hard or soft vascular sign was present. The data of the current study do not describe the indication for scans outside of blunt trauma, penetrating trauma and non-trauma; therefore, the lower yield rates may be due to scans being done without specific indications, as mentioned above.

As with the discrepancy between trauma and non-trauma limb scans, there was a large discrepancy in positive yield of CT scans of the neck.

Non-trauma neck pathology is often clinically obvious. Extra- and intrapharyngeal abscesses and masses can be seen, palpated and visualised on ultrasound. As these were the majority of non-trauma indications, the expected high yield was confirmed.

In trauma neck scans there are confounding issues. Despite clinical decision rules being practised in the ED in our study, patients who may not initially have required a cervical spine scan may later 
have needed one owing to ongoing neck pain. There may also be distracting injuries in polytrauma patients. In our ED, trauma patients who undergo CT scans of the head often simultaneously receive a CT scan of the neck. Such patients include those who were involved in high-velocity motor vehicle and pedestrian accidents, as well as those who fell from a height. However, it was noted that many patients receiving head CT scans for isolated blunt injury also received neck CT imaging. This may be a contributing factor in the low trauma CT neck yield rates, as indicated by Kulvatunyou et al. ${ }^{[25]}$ who found a positive rate of only $0.7 \%$ if a CT scan of the neck was done for isolated, direct blunt head injury. They noted that the positive neck findings were more likely in patients who were assaulted and then fell to the ground. Another confounding situation occurs when trauma patients are intoxicated. It is difficult to ascertain whether a decreased level of consciousness is owing to intoxication or head injury; these patients may then need a mandatory scan to rule out trauma as a cause. They may also receive a CT scan of the neck.

There are many contributing factors that need to be considered when making use of a CT scan, e.g. financial cost, radiation exposure, time and human resources, which may lead to criticism when a scan is negative. A radiologically negative scan does not necessarily mean that it was not indicated or clinically significant. This study has investigated the radiological positive yield of scans done, but not the clinical outcome of patients. In non-radiological, clinical practice, making use of special investigations may function as ruleout tests. Similarly, making use of highly sensitive troponins and a clinical decision rule, one may exclude myocardial infarction. ${ }^{[26]}$ If the troponin result is negative, it does not mean the test was unnecessary.

An argument can also be made for a positive scan that does not account for a patient's symptoms. For example, a patient undergoing a CT scan, after meeting criteria of the Canadian head CT rule for minor head injury, may be found to have a nasal bone fracture, which is not a clinically significant finding related to the patient's head injury. This is further emphasised by the implementation of the Canadian head CT rule for minor head injuries, where the CT rate decreased to $32 \%$, and the clinical positive rate was only 8\%. ${ }^{[27]}$ Hirano et al. ${ }^{[28]}$ refer to a scan with intracranial pathology as a 'positive' scan and one with an acute lesion that accounts for a patient's symptoms a 'true positive' scan. Positive radiological scan results v. clinically significant scan results were not assessed. The retrospective nature of this study did not permit the assessment of positive v. true positive scans.

Numerous ways to improve positive rates have been suggested and validated. Among these are clinical decision rules and protocols. Baghdanian et al ${ }^{[29]}$ reported that after implementation of a protocol for blunt abdominopelvic trauma, CT use decreased by $32.1 \%$. The decrease in CT use also increased the positive finding rate.

Many factors need to be taken into account regarding the decision to scan a patient. A radiologist cannot solely decide that a scan is not indicated if they are not directly involved with the patient or clinical team. However, the radiologist has a responsibility to facilitate the judicious use of a very important tool (the CT scan), taking cost, human resources and radiation into account. The clinical and radiological teams should share the decision in the best interest of the patient and not regard a negative scan as wasted effort or use of a resource.

\section{Conclusion}

This study was the first in SA to investigate the overall positive yield of CT scans done in an academic ED. This is an important indicator of the effective utilisation of CT, also as an effective tool. It answers the question, Are we ordering too few or too many CT scans in the ED?'. A higher utilisation rate is associated with a lower positive rate. With the higher positive yields in the data reviewed, it seems that too few scans are being done. It can, however, be argued that the higher positive yield rates are due to judicious use of CT scans in our resource-limited setting.

In a setting where the ratio of patient numbers to availability of CT scans is among the highest in the world, there is a need for guidelines or standards with regard to the acceptable utilisation rates and acceptable positive yields to monitor the judicious use of this important resource.

It is hoped that these findings will contribute to the development of such local guidelines and standards to ensure rational and effective clinical use of this modality to improve patient-centred outcomes.

Future studies to assess miss rates, morbidity and other patient outcomes should be done to ultimately answer the question of whether too few CT scans are being performed.

Acknowledgements. We are grateful to Dr Grace Rubin for her general support, input and assistance in facilitating data collection.

Author contributions. Both authors contributed substantially to conceptualisation, design, analysis and interpretation of data. KS drafted the initial version of the manuscript. Both authors revised and edited the manuscript. Final approval of the version to be published was made by both authors.

Funding. None.

Conflicts of interest. None.

1. Rigsby P, Pai R. Positivity rate of computed tomography of the abdomen and pelvis performed in the emergency department of a community based level 2 trauma center. 2013. http://c.ymcdn.com/sites/ www.aocr.org/resource/resmgr/imported/Rigsby.pdf (accessed 25 January 2018).

Schwartz DT. Counter-point: Are we really ordering too many CT scans? West J Emerg Med 2008;9(2):120-122.

3. Street M, Brady Z, van Every B, et al. Radiation exposure and the justification of computed tomography scanning in an Australian hospital emergency department. Intern Med 2009;39(11):713-719. https:// doi.org/10.1111/j.1445-5994.2009.01956.x

4. Kabongo JM, Nel S, Pitcher RD. Analysis of licensed South African diagnostic imaging equipment. Pan Afr Med J 2015;22(1). https://doi.org/10.11604/pamj.2015.22.57.7016

5hou JC, Zheng SW, Yu YX, et al. Trends in computed tomography utilization and association with hospital outcomes in a Chinese emergency department. PLOS ONE 2012;7(7):1-6. https://doi. org/10.1371/journal.pone.0040403

6. Oh HY, Kim EY, Cho J, et al. Trends of CT use in the adult emergency department in a tertiary academic hospital of Korea during 2001 - 2010. Korean J Radiol 2012;13(5):536-540. https://doi. crg 10.3348 sit.

7. Purg $\mathrm{S}$, Hu R, Quazi RR, et al. Physician and midlevel providers' awareness of lifetime radiation 7uri S, Hu R, Quazi RR, et al. Physician and midlevel providers' awareness of lifetime radiation
attributable cancer risk from commonly performed CT scan and its relationship to their practice attributable cancer risk from commonly performed CT scan and its relationship to ther
behaviour. Am J Roentgenol 2012;199(6):1328-1336. https://doi.org/10.2214/ajr.12.8581

8. Kindermann DR, McCarthy ML, Ding R, et al. Emergency department variation in utilization and diagnostic yield of advanced radiography in diagnosis of pulmonary embolus. J Emerg Med 2014;46(6):791-799. https://doi.org/10.1016/j.jemermed.2013.12.002

9. Oguz KK, Yousem DM, Deluca T, et al. Effect of emergency department CT on neuroimaging case volume and positive scan rates. Acad Radiol 2002;9(9):1018-1024. https://doi.org/10.1016/s10766332(03)80477-4

10. Prologo JD, Gilkeson RC, Diaz M, et al. CT pulmonary angiography: A comparative analysis of the utilization patterns in emergency department and hospitalized patients between 1998 and 2003. Am J Roentgenol 2004;183(4):1093-1096. https://doi.org/10.2214/ajr.183.4.1831093

11. Smith ZA, Postma N, Wood D. FAST scanning in the developing world emergency department. Smith ZA, Postma N, Wood D. FAST scanning in the developing wo
S Afr Med J 2010;100(2):105-108. https://doi.org/10.7196/samj.3821

2. Norman R, Matzopoulos R, Groenewald P, et al. The high burden of injuries in South Africa. Bull Worman R, Matzopoulos R, Groenewald P, et al. The high burden of injuries
Wealth Organ 2007;85(9):695-702. https://doi.org/10.2471/blt.06.037184

13. Wahlin RR, Ponzer S, Lövbrand H, et al. Do male and female trauma patients receive the same prehospital care? An observational follow-up study. BMC Emerg Med 2016;16(1):6. https://doi. org/10.1186/s12873-016-0070-9

14. Allard D, Burch VC. The cost of treating serious abdominal firearm-related injuries in South Africa. S Afr Med J 2005;95(8):591-594.

15. Sierink JC, Treskes K, Edwards MJ, et al. Immediate total-body CT scanning versus conventional imaging and selective CT scanning in patients with severe trauma (REACT-2): A randomised controlled trial. Lancet 2016;388(10045):673-683. https://doi.org/10.1016/s0140-6736(16)30932-1

16. Kirschner J, Shah K, Runde D, et al. Patterns in computed tomography utilization among emergency physicians in an urban, academic emergency department. Emerg Radiol 2014;21(6):577-581. https:// doi.org/10.1007/s10140-014-1237-x

17. Kameda T, Taniguchi N. Overview of point-of-care abdominal ultrasound in emergency and critical care. J Intens Care Med 2016;4(1):53. https://doi.org/10.1186/s40560-016-0175-y

18. Tsung JW, Tay ET, Elikashvili I. The effect of point-of-care ultrasonography on emergency department . Tsung JW, Tay ET, Elikashvili I. The effect of point-of-care ultrasonography on emergency department
length of stay and CT utilization in children with suspected appendicitis. Crit Ultrasound J 2014:6(1):1. length of stay and CT utilization in children with

19. Warwick JM, Sathekge MM. PET/CT scanning with a high HIV/AIDS prevalence. Transfus Apher Sci 2011;44(2):167-172. https://doi.org/10.1016/j.transci.2011.01.014 
20. Bent C, Lee PS, Shen PY, et al. Clinical scoring system may improve yield of head CT of non-trauma emergency department patients. Emerg Radiol 2015;22(5):511-516. https://doi.org/10.1007/s10140015-1305-x

21. Sinclair DE, Kovacs G, Hillis M. Cranial CT scans - emergency department utilization. J Emerg Med 1993;11(5):643-646. https://doi.org/10.1016/0736-4679(93)90325-2

22. Kelly AM, Kerr D Are too many head CT scans ordered in emergency departments? Emerg Med 2000;12(1):50-54. https://doi.org/10.1046/j.1442-2026.2000.00083.x

23. Ismail MS, Fathil SM, Soon BH, et al. Emergency head CT scan ordering and yield. J Emerg Med Trauma Acute Care 2008;8(3):151-155.

24. Monazzam S, Goodell PB, Salcedo ES, et al. When are CT angiograms indicated for patients with lower extremity fractures? A review of 275 extremities. J Trauma Acute Care Surg 2017;82(1):133-137. hatps/1/0iorg 10.10971 A 00000000001258

25. Kulvatunyou N, Friese RS, Joseph B, et al. Incidence and pattern of cervical spine injury in blunt assault: It is not how they are hit, but how they fall. J Trauma Acute Care Surg 2012;72(1):271-275. https://doi.org/10.1097/ta.0b013e318238b7ca
26. Ishak M, Ali D, Fokkert MJ, et al. Fast assessment and management of chest pain without ST-elevation in the pre-hospital gateway: Rationale and design. Eur Heart J Acute Cardiovasc Care 2015;4(2):129136. https:///oi.org/10.1177/2048872616687116

27. Stiell IG, Wells GA, Vandemheen $K$, et al The Canadian CT head rule for patients with minor head injury. Lancet 2001;357(9266):1391-1396. https://doi.org/10.1016/s0140-6736(00)04561-x

28. Hirano LA, Bogardus ST, Saluja S, Leo-Summers L, Inouye SK. Clinical yield of computed tomography Hirano LA, Bogardus ST, Saluja S, Leo-Summers L, Inouye SK. Clinical yield of computed tomography
brain scans in older general medical patients. J Am Geriatr Soc 2006;54(4):587-592. https://doi brain scans in older general medical
org $/ 10.1111 / j .1532-5415.2006 .00692$

29. Baghdanian AH, Baghdanian AA, Armetta A, et al. Effect of an institutional triaging algorithm on the use of multidetector CT for patients with blunt abdominopelvic trauma over an 8-year period. Radiology 2016;282(1):84-91. https://doi.org/10.1148/radiol.2016152021

Accepted 2 October 2017 